\title{
The AODV Extension Protocol Named AODV_SPB
}

\author{
Amina Guidoum \\ Electronic Department, Sidi Bel Abbes, 22000, Algeria \\ E-mail: guidoum_amina@ @otmail.fr \\ Aoued Boukelif \\ Electronic Department, Sidi bel Abbes, 22000, Algeria \\ E-mail: abouklif@yahoo.fr
}

Received: 10 February 2019; Accepted: 24 February 2019; Published: 08 March 2019

\begin{abstract}
An unbalanced traffic load distribution leads to a degradation of network performance; most of nodes in the network are heavily loaded, resulting in a large queue, a high packet delay, and high energy consumption. The optimization of load balancing to avoid congestion has been the subject of many researches in recent years. Many authors have proposed different solutions to anticipate the failure of route in Manets by adding a function that predicts the failure of the links to distribute the traffic load on all nodes of the network. In this paper we propose an extension to the AODV-balanced protocol named AODV_SPB « ad hoc on demand distance vector with stable path, less congested with load balancing », which looks for a stable and less overloaded path.A comparative study is done under the NS2 simulator with AODV and AODV-SPB. This last protocol shows its effectiveness with respect to the two protocols cited in terms of; overload, delivery rates of packets and the average of delay with 4 simulation scenarios.
\end{abstract}

Index Terms-AODV_SPB, Manets, Link Failure Prediction, AODV_balanced, AODV.

\section{INTRODUCTION}

Ad hoc networks provide new applications but the technical difficulties encountered show that layered architectures are not suitable for its wireless networks [1].

As in the case of mobile networks, the integration of multimedia services into ad hoc networks has attracted considerable interest in recent years. Noting that the problems posed in the particular context of ad hoc networks are different and more complex than those encountered in the wired world. For this purpose, studies have been made to improve the performance of networks.

The mobility model shows the movement of real moving nodes that change speed and direction over time. The mobility model that accurately represents the characteristics of mobile nodes in an ad hoc network is the key to examine the usefulness of a given protocol in a particular type of mobile scenario. On the basis of mobility characteristics, different models of mobility exist such as in [2]

The main purpose of routing protocols is to establish and maintain routes so that the data are properly delivered to the destination. Different protocols have been created and classified according to various researches [3], and comparisons made on each protocol class.

In the last years, several ad hoc routing protocols have been proposed, either by modifying existing routing protocols or by developing new routing approaches. They are classified As follows: proactive, reactive or hybrid. In proactive routing algorithms, each node in the network must have maintained routing information and stored them in routing tables. In the case where a reactive routing protocol is used, the routes are created on demand. It means that; a new traffic is launched according to the request-response principle. When a node wants to initiate a communication with another node, it starts a route discovery process. Once the route is found, it is maintained by a route maintenance procedure until the route is no longer used. An example of the reactive routing protocol is: AODV [4]. They all aim to find the shortest path between a source and a destination taking into account the state of the node and the configuration of the network when a route is requested. In addition to reactive and proactive routing protocols, there is a family of routing protocols that mixes both is called 'hybrid'.

The routing mechanism is paramount in MANETS. It is responsible for distributing the load between source and destination. Also, it is a way to find an optimal path for routing data packets.

Since ad hoc networks do not have a centralized control for load distribution, balancing becomes an important issue. A combination of a load balancing and a mathematical method of anticipating the failures of paths has been proposed.

Congestion is the main cause of link failures due to excessive load on the network. Therefore, an excessive load on the node results in saturating the full memory buffer which causes the removal of packets.

The distribution of the load is the solution to avoid congestion in the network. If the load is well balanced or distributed across the network, it will reduce the end-to- 
end delay and it will improve the delivery rate [5].Traffic Load Balancing aims at moving traffic from overloaded areas to less loaded areas so that, the network gets the best performance.

When the requirements become greater than the maximum capacity of the communication link; for example, multiple hosts attempting to access a shared medium; congestion occurs in the network.

When the number of nodes increases [6], congestion degrades the performance of this network such as throughput, number of packets lost, and energy conservation. Most protocols do not take into consideration overload and congestion. This problem will encounter that a strong overload, more communication and processing are needed to find a new path.

The rest of the paper is organized as follows: Section 2 describes the Literature review. In Section 3 the proposed protocol is presented. In Section 4 the performances is evaluated and compared via simulation.

\section{RELATED WORKS}

Several approaches have been proposed in order to solve the problem of congestion and mobility that causes paths failures and loss of information. Therefore, the performance of MANETS is degraded. To balance the network traffic load there are different ways: first, it is based on link state. Second, it is based on traffic. Third, it is based on the combination of both. [7].

In this paper, the authors SB Hacene, M.aliCherif and MK Feraoun propose [8] a new protocol named LBPAODV. It is based on the combination of two new metrics: link quality and MAC_OVERHEAD. A network mechanism; Inter layer; exists between the MAC layer and routing layer by exchanging messages. If the link failure is due to mobility or congestion, the variable HIGH RSS(received signal strength) will be used to distinguish between the two situations.

The proposed mechanism for congestion control in LO-PPAOMDV implemented an inter-layer approach that follows the RSS of packets received from each neighbor node to know when a node is close enough for transmission. The failure prediction technique of link is based on the Lagrange interpolation to estimate when an active link fails. For the MAC extension, when a node tries to communicate with a neighbor node but this communication fails after several attempts, the MAC layer sends error to this layer. The protocol interprets that the neighbor node is not near. So, the failure of the communication is due to the mobility.

According to this paper, in a scenario without mobility the failure of communication can arise. Bisengar, M. Rziza and M. Ouadou proposed in [9] an AM_AODV protocol "adaptive mobility aware ad hoc on demand distance». This protocol is derived from the AODV protocol in order to reduce mobility, congestion and minimize control messages to find a more stable path. This path will be chosen according to distance, weight, relative speed, queue length and number of hop mobility model ".Two ray ground "[10] has been used because it gives more prediction of the signal strength at a long distance than the other models. The algorithm of route discovery is modified by means a cost is added to the RREQ packet containing the function of the weight.

Sujuta Mallapur in [11] describes an approach based on path failure prediction and a new paths maintenance mechanism. When a node starts the path search to route the data packets, the path discovery process is triggered. The source sends a RREQ packet. The receiver node calculates the received power of the packet and compares it to a threshold value. If it is inferior, this packet will be deleted and sends an ALERT packet to the sender node of the packet. This node cancels the path. The same thing for the packets of data:

The received power of the packet at the receiving node is calculated. If it is inferior a RRER packet is sent to the source. The path maintenance mechanism is implemented either to repair the failing path or to find a new route when all the paths fail.

SH Boukli, A. Lehireche and A. Meddahi in [12] proposed several schemes to improve performances of the routing protocol by using the prediction of mobility and minimization of unnecessary messages and repairs the paths before their failure. The formula of the Lagrange interpolation calculates the power. So, the node; after receiving the signal powers with their times; approximates the value of the power and compares it to the threshold .If it is lower an alert message is sent to the predecessor node. Then, it starts the reparation process of the link in order to find an alternate path to the destination using the link to the node that sent the alert message.

In [13] A.Boukerche and L. Zhang present a preemptive routing protocol for ad hoc mobile networks that describes a pre-emptive technique launches path discovery in advance and warns the source before the path fails by exchanging HELLO messages "the pingpong process». When the value of the received packet power is lower than a threshold value, the node sends to its neighbors a HELLO message called ping. The neighboring nodes must respond with a pong packet in a delay. If the node does not receive the pong message an alert message is sent to the source. Once the alert message is received, the source node starts rediscovering. The discovery in advance is accomplished by gathering information about the active links.

In [14] Mu-Qing WU, Yan ZHEN, Da-peng WU propose a protocol called "ODLDE" which estimates the lifetime of links by the mean intercept method with the use of the reference points. It also estimates the continuous reception of the signal strength by the Newton interpolation method. The results of the simulation showed that the lifetime of the link can be predicted independently of the mobility model. The operating principle of this protocol is derived from the AODV protocol that each node constructs a table indexed by the addresses of the neighboring nodes, the lifetime of the link between the node and its neighbor. The lifetime of the Link is computed and saved in the table using the lifetime estimation of the previous link. 
In this paper [17] Anita Yadav ,Y. N. Singh and R. R. Singh Proposed the AODVLP protocol which is an improvement of the AODV protocol, they used "polynomial interpolation" to estimate the link failure time (td),

The nodes estimate the break time of the link and warn other nodes of link interrupts on the route; This is done by estimating the time at which the signal strength received from the data packets will be below a threshold power.

And the polynomial interpolation of Newton is defined by (Pr) which is the threshold of the power of the signal at the time tp, that tp which will be predicted. The power of the threshold signal Pr is the minimum power that can be received by the device. the disadvantage of estimating the time td :many control messages sent, which encumbers and causes overload in the network, which will not be effective in real-time applications.

\section{THE PROPOSED AODV_SPB}

Since ad hoc networks do not have centralized control for traffic load distribution, balancing traffic load becomes one of the most important subjects.

For the protocols $[5,15]$, a scheme has been proposed to distribute the traffic load. The algorithm of the AODV protocol has been modified in which a new cost function is added to the route discovery phase based on the load Traffic of the active path. The selected path by the destination is the least overloaded with fewer hops. Other contributions such as a procedure to avoid congested nodes are added.

This new protocol has shown its effectiveness compared to AODV in terms of quality of service. It improves the delivery rate, minimizes the end-to-end delay and reduces the overload according to the results of the simulation. Because of its characteristics, AODV_balanced protocol [15] was used in this paper to be enhanced.

An improvement has been proposed to "AODV_balanced" protocol [15] to anticipate and predict route failure. The new protocol called AODV_SPB (ad hoc on demand distance vector with stable path, less congested and traffic load balancing) is a combination of traffic load balancing technique and route failure prediction scheme.

Given the instability of links and the route failures due to frequent changes in the topology of the ad hoc network caused by the mobility of the nodes, we opted for the path failure prediction. As a polynomial interpolation, Newton polynomial interpolation (NPI) is a tool can continuously approximate the signal strength with limited sampling points. Taking as parameters the powers of the received packets with their measurement times, the formula is as follows:

$\mathrm{N}(\mathrm{x})$ represents the approximated signal strength.

$$
x_{0}, x_{1} \ldots ., x_{n} \in[a, b] \text { Represents the current }
$$

sampling instants,
$Y_{0}: f\left(x_{0}\right), y_{k}: f\left(x_{k}\right), f(x):$ The received signal strength.

The NPI is defined as follows:

$$
\begin{aligned}
& \mathrm{N}(\mathrm{x})=\left[\mathrm{y}_{0}\right]+\left[\mathrm{y}_{0}, \mathrm{y}_{1}\right]\left(\mathrm{x}-\mathrm{x}_{0}\right)+\left[\mathrm{y}_{0}, \mathrm{y}_{1}, \mathrm{y}_{2}\right]\left(\mathrm{x}-\mathrm{x}_{0}\right)\left(\mathrm{x}-\mathrm{x}_{1}\right)+ \\
& \ldots \ldots+\left[\mathrm{y}_{0}, \ldots, \mathrm{y}_{\mathrm{k}}\right]\left(\mathrm{x}-\mathrm{x}_{0}\right) \ldots\left(\mathrm{x}-\mathrm{x}_{\mathrm{k}-1}\right)
\end{aligned}
$$

The node after receiving the signal powers with their times approximates the value of the power $\mathrm{N}(\mathrm{x})$ and compares it with the threshold value,

If it is less than the threshold a RRER message is sent to the predecessor node, and starts the link repair process for find an alternative path to reach the destination using the link to the node that sent the alert message. (The procedure of the local repair is triggered; this function is defined in the route maintenance phase of the AODV protocol.):

Pseudo code for routing algorithm:

Precondition: The source node broadcasts the RREQ packet to the neighboring nodes to discover a new route:

If (the receiving node of the RREQ packet finds that the identifier of the request already exists)

\{Delete the RREQ packet\}

Else \{Initiating the procedure for checking the load status of the node

If (the node is overloaded)

\{Delete the RREQ packet\}

Else

\{ Call for the cost function and add the value of the cost to the RREQ

Create an entry for the destination source pair

Save the previous hop for that entry.

Go to: pseudo code 2 MAX)

Broadcast the RREQ packet while (RREQ retries < = \}

Pseudo code 2:

Precondition: The node receives the powers and the measurement times of the received packets

If (the index of the sender exists in the list of nodes)

\{Inserting Power and Time into the Power List

$$
\text { Else }
$$

Create a new cell for the node (the node address will be added) that has a pointer to the list of powers whose power and time will be inserted

If (the power list size reaches the max)

\{Prediction is triggered by calling Newton Polynomial Interpolation $\}$

If $(\mathrm{N}(\mathrm{x})<($ threshold $))$

\{Call local Repair ()

The index of the node will be deleted as well as its powers and its corresponding times 


\section{SIMULATION AND EXPERIMENT}

To evaluate performances of the routing protocols, we chose the network simulator NS2 [16]. It is an open source widely used for research in the field of MANETS. Protocols extensions can be added to this simulator. An analysis of the results is possible as shown in the table1, figure 1 , figure 2 , figure 3 .

The metrics for evaluating and comparing the performances of; the AODV, AODV-balanced and AODV-SPB routing protocols, considered in this study; are the overload, the rate of packets delivered, and the average delay of the packets according to the pause time under four different scenarios [15].

Table 1. Simulation Parameters

\begin{tabular}{|l|l|}
\hline Simulation parameters & values \\
\hline Network & MANET \\
\hline Propagation model & Two ray ground \\
\hline Simulation tool & NS2 \\
\hline Simulation Time & $900 \mathrm{~s}$ \\
\hline Simulation area & $1500 \times 300 \mathrm{~m}$ \\
\hline Number of nodes & 50 \\
\hline Mobility pattern & Random waypoint \\
\hline Source type & CBR \\
\hline Routing protocols & $\begin{array}{l}\text { AODV }, \text { AODV_balanced, } \\
\text { AODV-SPB }\end{array}$ \\
\hline Pause time & $\begin{array}{l}0,30,60,120,300,600, \\
900 .\end{array}$ \\
\hline Rate & 4 packets/second \\
\hline Packet size & 512 octet \\
\hline Threshold & -81 B \\
\hline Scenarios & $\begin{array}{l}\text { SCENARIO1 : max } 10 \\
\text { connections } \\
\text { SCENARIO2 : max 20 } \\
\text { connections } \\
\text { SCENARIO3 : max 30 } \\
\text { connections } \\
\text { SCENARIO4 : max 40 } \\
\text { connections }\end{array}$ \\
\end{tabular}

\section{Discussion:}

\section{Overhead}

Overhead represents the number of routing packets transmitted per data packet delivered at the destination.

The figures represent the results obtained from each scenario concerning the Overhead metric and the X-axis: pause time.

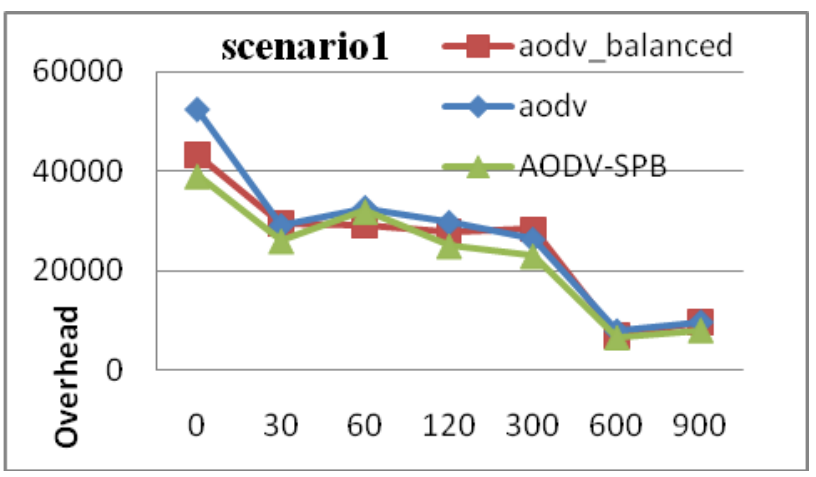

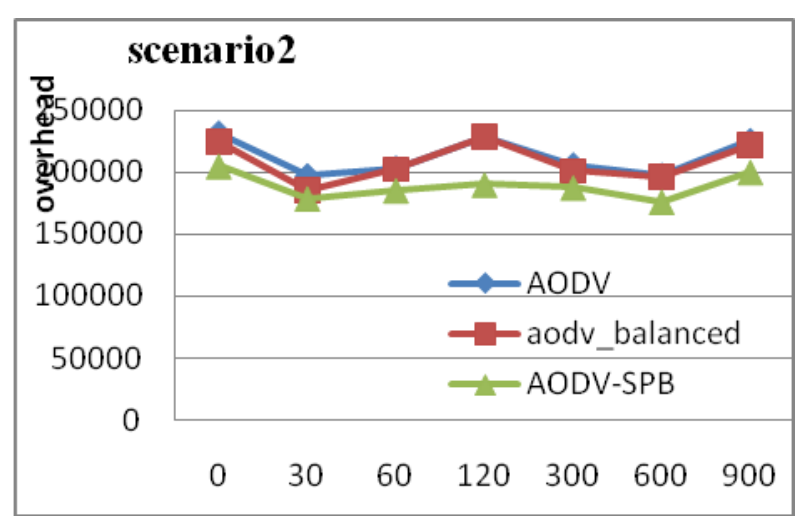
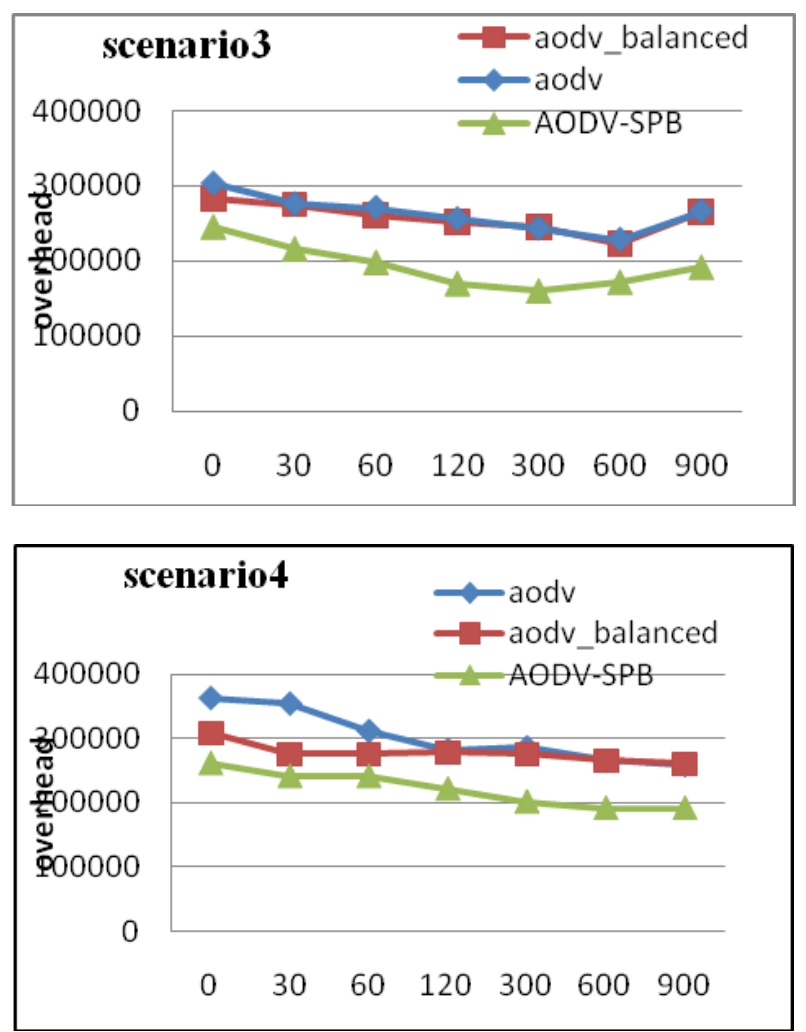

Fig.1. Protocols Performances Evaluation (4scenarios): Overhead

The curves in figure 1 show; in term of overload for the three protocols AODV-BALANCED, AODV-SPB and AODV; deterioration in a high-mobility environment (pause time $=0$ ). Compared to the standard protocol (AODV), the modified protocol AODV-SPB and AODVBALANCED give very good results for all pause time values. It is due to the load balancing technique which avoids congested nodes and chooses the least overloaded routes.

\section{Delivery Ratio:}

Delivery ratio: is the ratio of the number of data packets received by the destination and the number of data packets sent by the source.

The figures represent the results obtained from each scenario concerning the Delivery ratio metric and the $\mathrm{x}$ axis: pause time 

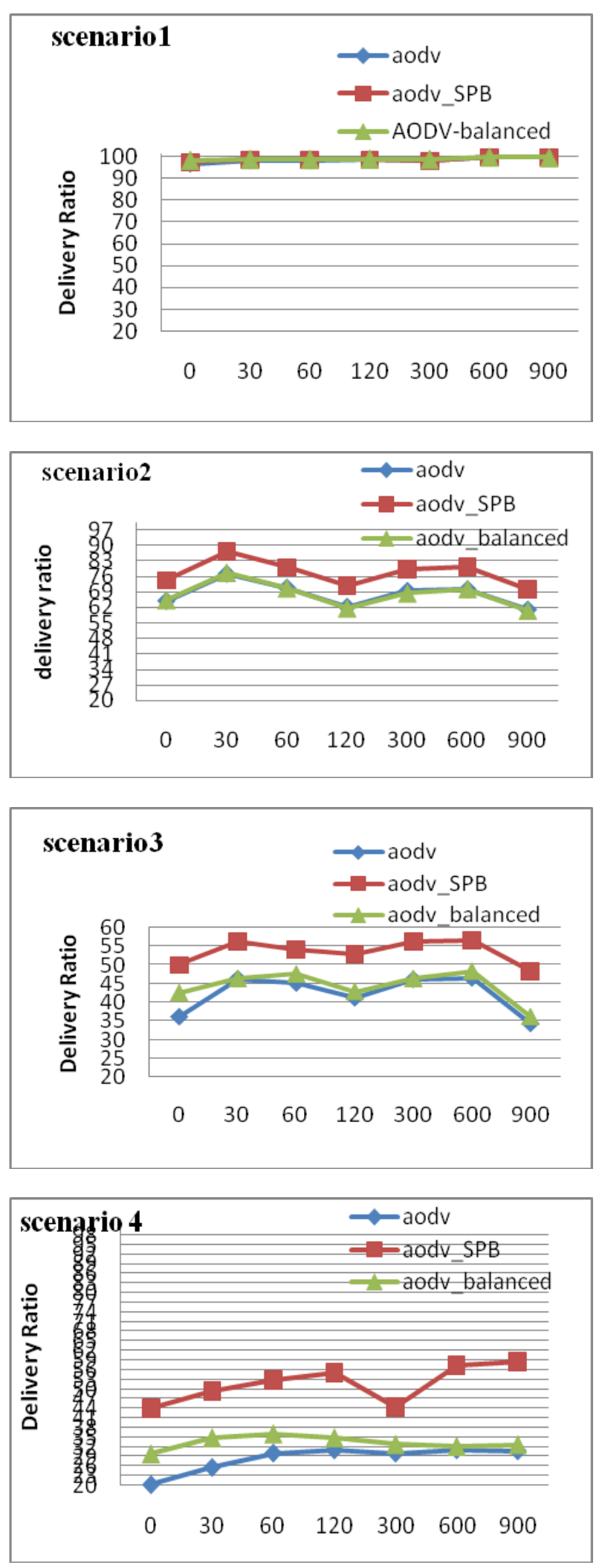

Fig.2.Protocols Performances Evaluation (4scenarios): Delivery Ratio.

From figure 2, it can be seen that the AODV_SPB protocol ensures an improvement in the rate of the delivered packets. It reaches $45 \%$ in the worst case scenario (high mobility environment) and $97 \%$ for a stable environment. This is due to the combination of the route failure prediction mechanism .Therefore, fewer packets are lost and the delivery rate represents the number of packets received by the number of packets sent, therefore this rate has been improved.

\section{Average Delay}

The average delay is defined as is the average time between sending a packet and receiving it.

End-to-end delay includes routing delay and other miscellaneous delays, such as, transmission delay, propagation delay, and delay of the queue.

The figures represent the results obtained from each scenario concerning the Average delay metric and the $\mathrm{x}$ axis: pause time.

In the four scenarios of figure 3 , the results of the simulation show that the curves decrease when the delay depends on the mobility. For a high mobility the AODVSPB protocol minimizes the delivery time of the packets much more than the other two protocols AODV and AODV_BALANCED.
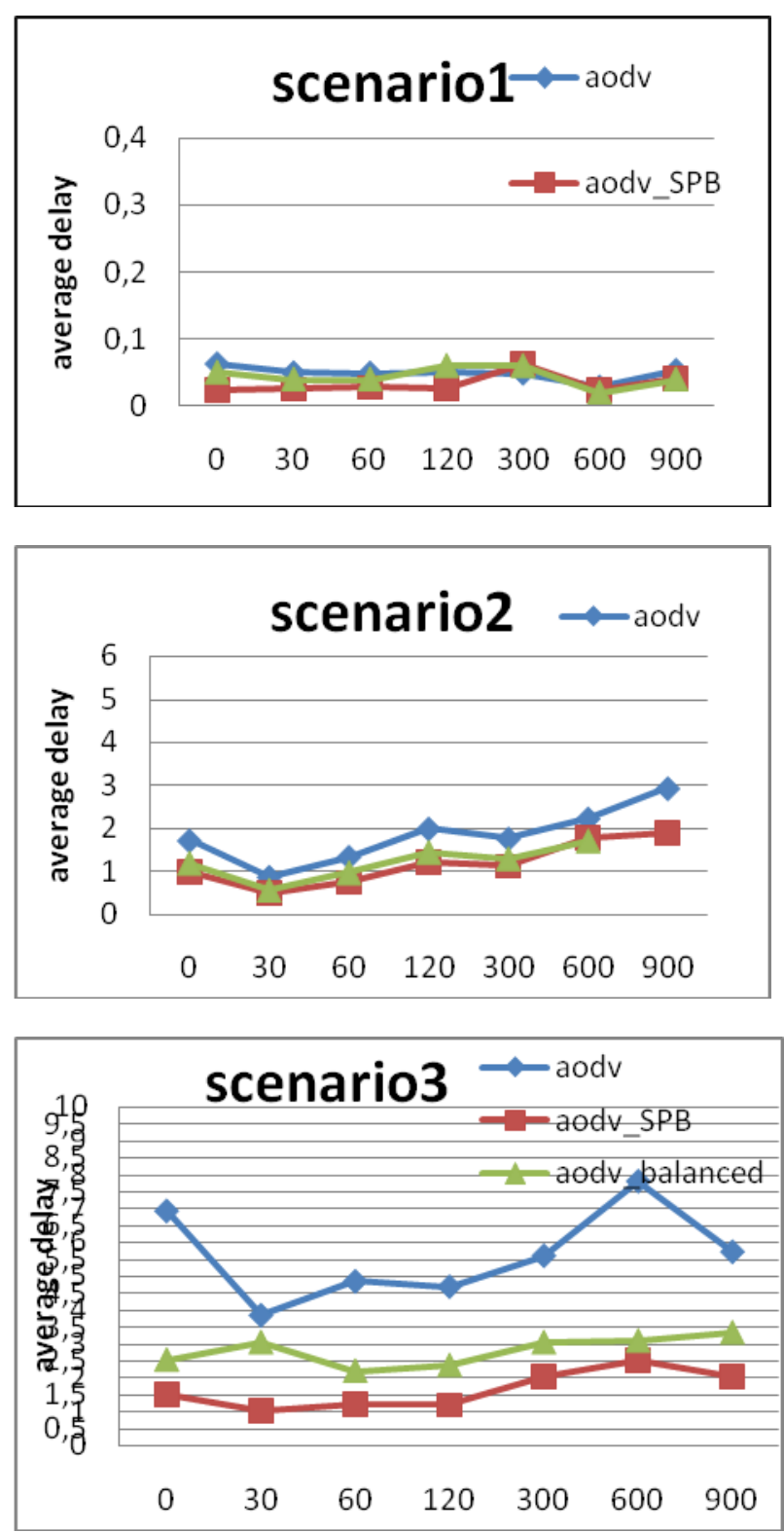


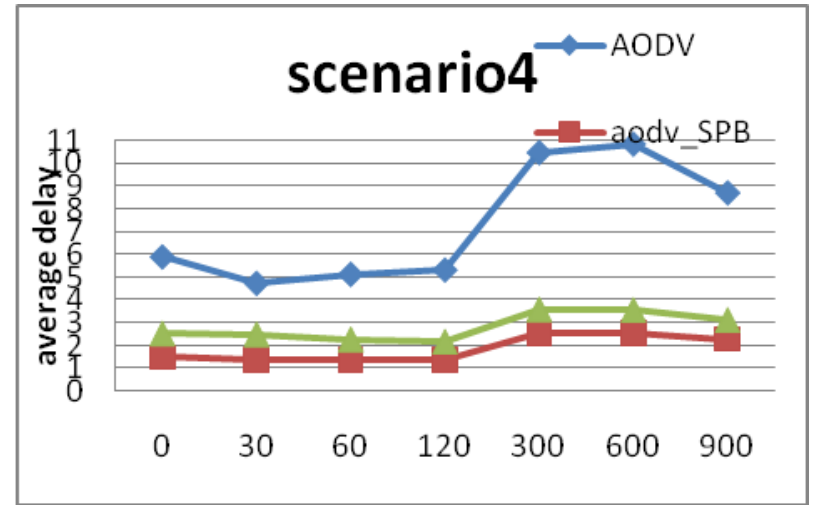

Fig.3. Protocols Performances Evaluation (4scenarios): Average Delay.

In summary, the results of the simulations carried out show that the protocol AODV_SPB performs well compared to the standard version of the AODV protocol and the AODV_BALANCED protocol in a low or high mobility environment. Since it is a hybrid protocol which combines traffic load distribution technique with path failures prediction scheme.

\section{CONCLUSIONS}

The experimental results obtained using the NS-2 network simulator confirm that the proposed AODV-SPB protocol combines the stability of the path by predicting link failures with the efficiency of routing based on congestion avoidance.

As future work we can implement this algorithm to the different protocols of the ad hoc networks for an optimal routing with load balancing of the traffic and more stable paths which improves the quality of service.

\section{REFERENCES}

[1] R. Chandran, Shanmugavel, "Received Signal Strengthbased Cross-layer Designs for Mobile Ad Hoc Networks", IETE Technical Review, Vol. 25, no. 4, pp. 192-200, Jul. 2008.

[2] D. Smita, S. Anuja, "Classification of MANET A Review”, INT J INNOV ENG TECH, Vol .5, pp. 300305,Apr. 2015.

[3] B. azzedin, T. begumhan, A. nevin, Z. Mohammad, "A Routing protocols in ad hoc networks", Comput Netw, Vol. 55, pp. 3032-3080, May.2011.

[4] C. Perkins, E. Belding-Royer, Das, "Ad hoc On-Demand Distance Vector (AODV) Routing”, RFC 3561, pp.7-24, 2003.

[5] A. Guidoum and A. Boukelif, "Optimization of AODV routing protocol in mobile ad-hoc network by introducing features of the protocol LBAR", In Proc. ECCOM '14, Geneva, sweetzerland, 2014, pp. 166-171.

[6] K. Vishnu, S. Sarita, "Mobile Agent Based Congestion Control Using Aodv Routing Protocol Technique for Mobile Ad-Hoc Network", Int $J$ of Wireless \& Mobile Networks, Vol .4, pp. 300-314, Apr.2012.

[7] J. Sunsook and N. Hundewale, A. Zelikovsky, "Energy efficiency of load balancing in MANET routing protocols", In Proc. SNPD-SAWN, 2005, pp. 484-490

[8] M. cherif, K. Faraoun, S. Boucli, "Link Quality and MAC-Overhead aware Predictive Preemptive Multipath Routing Protocol for Mobile Ad hoc Networks", Int J Commun Networks INF Security, Vol .5, pp. 210-218, Dec.2013.

[9] A. Bisengar, M. Rziza, M .Ouadou, "Queue Length and Mobility aware Routing Protocol for Mobile Ad hoc Network", Int J Commun Networks INF Security, Vol.4, pp. 207-211, Dec. 2012.

[10] T. Rappaport, "Wireless communications. Principles and practice", Prentice Hall, 2nd ed, 2002.

[11] S. Mallapur, "Predictive Preemptive Ad Hoc on-Demand Multipath Distance Vector Routing Protocol", Int J of Computer Science \& Emerging Technologies, Vol.1, pp. 155-160, Aug.2010.

[12] S. Boukli, A. Lehireche, A. Meddahi, "Predictive Preemptive Ad Hoc On-Demand Distance Vector Routing”, Malaysian J of Computer Science, Vol. no. 19, pp.189-195. Feb.2006.

[13] A. Boukerche, L. Zhang, "A performance evaluation of a pre-emptive on-demand distance vector routing protocol for mobile ad hoc networks", wirel commun mob COM, Vol. 4, pp. 99-108, Feb.2004.

[14] W. MuKing, Z. Yan, W. Dapeng, "Reliable routing mechanism based on neighbor stability for MANET", it the J of China Universities of Posts ,Vol.16, pp.33-39, 2009.

[15] A Guidoum, A boukelif, "Novel Approach to Enhance Quality of Service by Distributing the Load of Traffic in Manets", WSEAS Transactions on Computers,Vol.16, pp.320-325, 2017.

[16] K. Ruoshan, "The Simulation for Network Mobility Based on NS2", In Proc. ICCSSE, 2008, pp. 1070-1074.

[17] Y Anita, N Singh, R Singh, "Improving Routing Performance in AODV with Link Prediction in Mobile Ad hoc Networks", Wireless Pers Commun, 83, pp.603-618., 2015.

\section{Authors' Profiles}

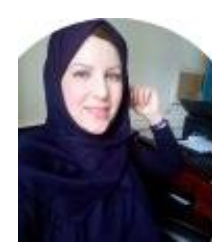

Guidoum Amina was born in Sidi bel abbes, Algeria in 1987. She graduated in Electronics, where she received the Master in 2012 and Ph.D degree in Electronic (Network Architecture \& Multimedia) at the faculty of technology department electronics in sidi bel Abbes University, 2019.

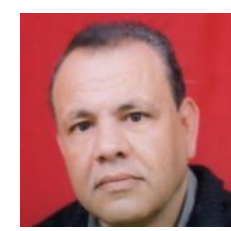

Phd degree in image processing, Professional speaker, author and consultant in educational sciences, ICTs and digital pedagogy. Professor of Electrical Engineering and IT's, Djillali Liabes University of Sidi Bel Abbes. 
How to cite this paper: Amina Guidoum, Aoued Boukelif,"The AODV Extension Protocol Named AODV_SPB", International Journal of Computer Network and Information Security(IJCNIS), Vol.11, No.3, pp.15-21, 2019.DOI: 10.5815/ijcnis.2019.03.03 